\section{Fellowship an der Plastisch Chirurgischen Abteilung des Chang Gung Memorial Universitätsspitals, Taipei-Linkou, Taiwan}

\author{
Microvascular Surgery Fellowship at the Department of \\ Plastic and Reconstructive Surgery, Chang Gung Memorial \\ University Hospital, Taipei-Linkou, Taiwan
}

\section{Taiwan \\ $\nabla$}

Taiwan ist ein Inselstaat mit 23 Mio. Einwohnern, der in etwa ein Drittel der Fläche von Österreich umfasst und liegt geografisch östlich von China, südwestlich von Japan und nordöstlich von Hong Kong. Das Bruttoinlandsprodukt (BIP, in English GDP $=$ Gross Domestic Product) per capita in Taiwan beträgt 38000 US\$ (Stand 2013, CIA World Factbook, www. cia.gov). Weiters ist Taiwan Headquarter von internationalen Firmen wie zum Beispiel Acer, Asus, HTC, Evergreen, Giant, Tern, Dahon, Foxconn und vielen mehr. Die Hauptstadt Taipei ist der Sitz des zweithöchsten Wolkenkratzers der Welt Taipei 101 (www.taipei-101.com.tw). Er gilt als Symbol der Evolution der Technologie und der asiatischen Tradition und steht wie ein Bambusbaum in der 8 Mio. Metropole. Das National Palace Museum (www.npm.gov.tw), das einzige Museum der traditionellen chinesischen Kultur mit 696000 Ausstellungsstücken der letzten 5000 Jahren, befindet sich in Taipei, wo aufgrund der hohen Anzahl der Ausstellungsstücke, nur jeweils 1\% gleichzeitig ausgestellt werden können.

\section{Chang Gung Memorial Universitätsspital (CGMH)}

In 1974 wurde die Chang Gung Memorial Foundation gegründet, eine Non-profit Organisation mit der Vision die beste Gesundheitsinstitution weltweit zu werden. Sie steht unter privater Führung der „Formosa Plastica“, der größten Industrieholding Taiwans. Derzeit verwaltet Chang Gung Memorial Foundation 7 Spitäler mit insgesamt 9980 Betten, ein Pflegeheim (349 Betten) und ein Health Village (706 Wohneinheiten) ( $\bullet$ Abb. 1).

Das Chang Gung Memorial Universitätsspital (CGMH) in Linkou wurde in 1976 eröffnet (www1.cgmh.org.tw/branch/lnk/ e/index.aspx) und ist derzeit das größte Spital der Welt mit 3715 Betten und ca. 10000 Angestellten, gefolgt von West China Hospital of Medical Sciences, Sichuan University in China und Chris Hani Baragwanath Spital in Soweto, Johannesburg Südafrika. CGMH in Linkou hat aufgrund der nahen Lage zur Hauptstadt Taipei, zum internationalen Flughafen (TPE) und dem naheliegenden Industriegebiet, ein sehr großes Einzugsgebiet. Es werden täglich ca. 14000 ambulante Patienten und ca. 700 Notfallspatienten behandelt und etwa 200 Operationen im Zentral-OP mit 67 Tischen durchgeführt.

\section{Department of Plastic \& Reconstructive Surgery $\nabla$}

Das Department für Plastische und Rekonstruktive Chirurgie in CGMH Linkou (www1.cgmh.org.tw/intr/intr2/c3250/ en/profile.htm) wurde 1976 unter der Leitung von Professor Dr. Samuel Noord- hoff mit der Vision: „Refine, Innovate and Aim High" gegründet und ist in 6 Abteilungen unterteilt: Ästhetische Chirurgie, Allgemein-Plastische Chirurgie, Kraniofaziale Chirurgie, Mikrochirurgie, Trauma Chirurgie und Verbrennungschirurgie ( $\bullet$ Abb. 2). Mit ca. 50 OberärztInnen und 20 AssistenzärztInnen und einer Normalbettenstation mit 51 Betten und weltweit der einzigen Intensivstation für mikrochirurgische Fälle mit 24 Betten werden jährlich ca. 900 mikrovaskuläre Operationen durchgeführt. Seit 1985 wurden ca. 17000 freie Lappenplastiken, 4000 Replantationen und 2200 Plexus Rekonstruktionen an der Abteilung durchgeführt.

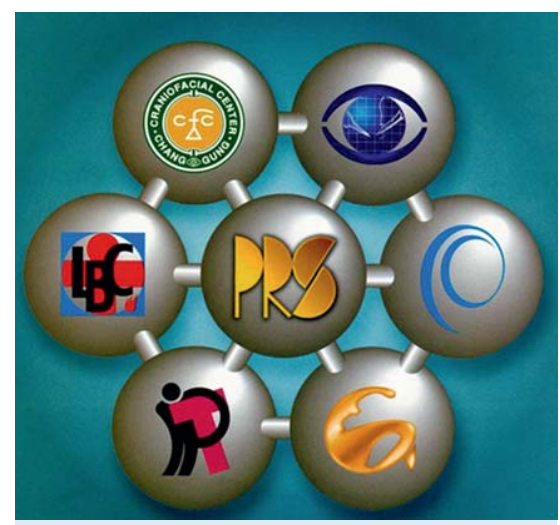

Abb. 2 Logo des Departments für Plastische und Rekonstruktive Chirurgie in CGMH Linkou und dessen klinischen Abteilungen: Ästhetische Chirurgie, Allgemein-Plastische Chirurgie, Kraniofaziale Chirurgie, Mikrochirurgie, Trauma Chirurgie und Verbrennungschirurgie.

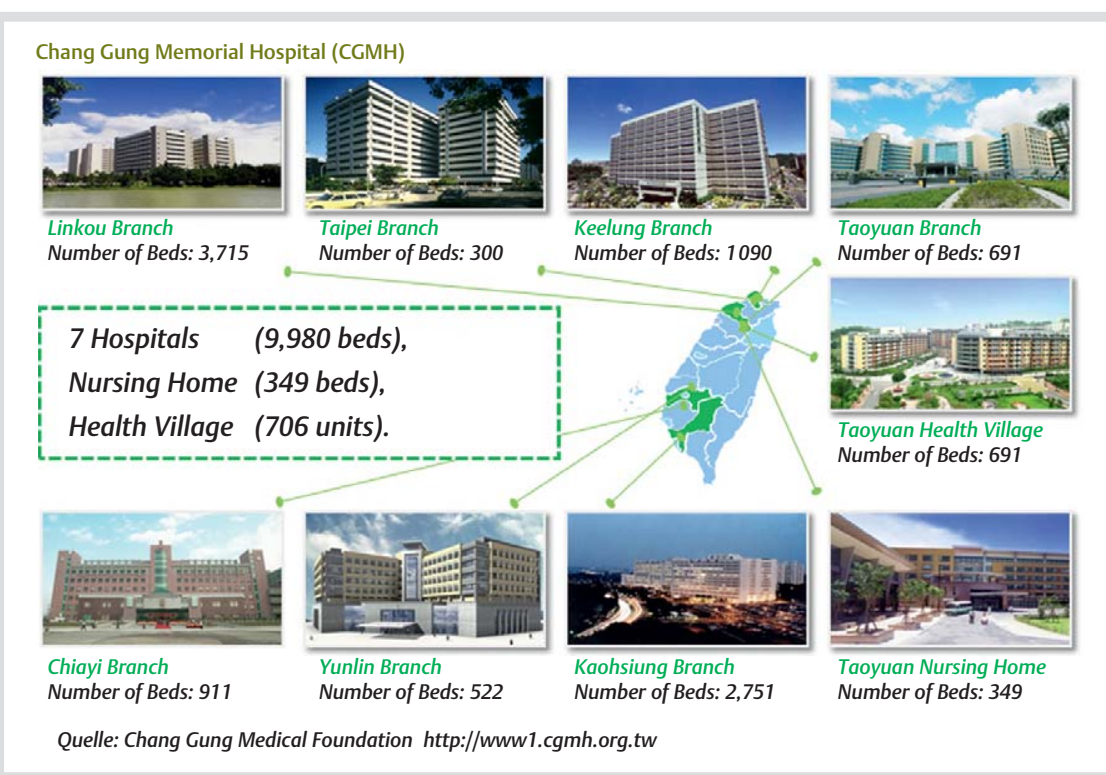

Abb. 1 Chang Gung Memorial Foundation 2013: 7 Spitäler (9980 Betten), ein Pflegeheim (349 Betten) und ein Health Village (706 Wohneinheiten) (Quelle: http://www1.cgmh.org.tw). 


\section{Microvascular Surgery Fellowship $\nabla$}

1983 wurde von Prof. Dr. Fu-Chan Wei das Visiting- und Fellowship-Programm initiiert (www.changgungmicro.com). Seither wurden in diesem einjährigen mikrovaskuläre Fellowship ca. 200 Fellows ausgebildet und es wurden ca. 1000 Visiting Scholars registriert ( $\bullet$ Abb. 3 ). Es sind jährlich ab 01. Juli 6 Plätze für das Fellowship zu besetzen, Einreichfrist für den Antrag ist jeweils der 31. Juli des Vorjahres. Das Fellowship ist eine „Hands-ON“ Ausbildung mit dem Schwerpunkt in der Mikrochirurgie. Die Fellows werden jeden 2. Monat einem Oberarzt zugewiesen, um die Techniken des jeweiligen Mentors zu erlernen. Mit dieser Zuteilung sind die Fellows wöchentlich 1-2× in der Ambulanz und $2-3 \times$ im Operationssaal. Zusätzlich

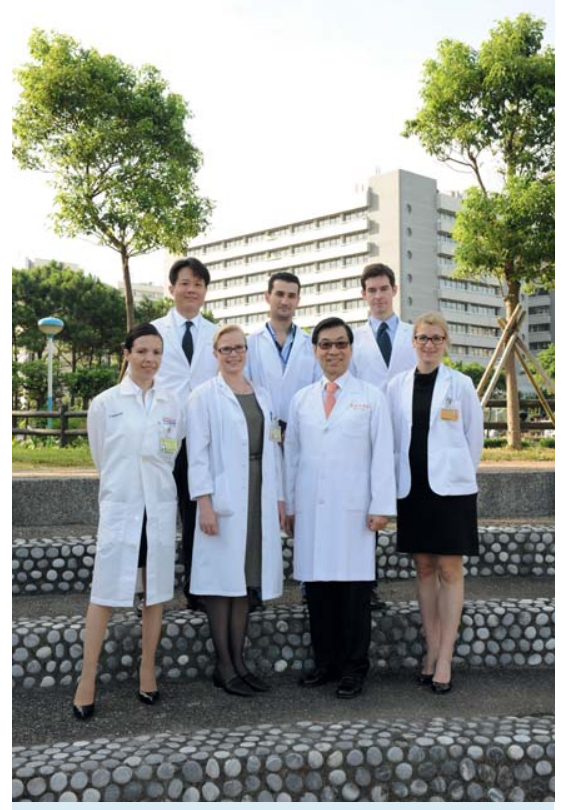

Abb. 3 Gruppenfoto 2012 Fellows mit Prof. Dr. Fu-Chan Wei; oben links: Chieh-Han John Tzou, Nidal Al Deek, Paolo Fanzio; unten links: Katerina Anesti, Heli Kavola und Leila Kolios.

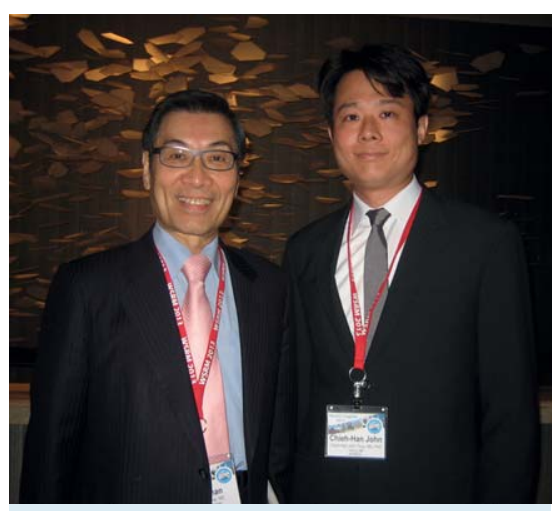

Abb. 4 Foto mit Prof. Dr. Fu-Chan Wei (2013 WSRM Meeting, Chiacgo, USA). zu den zahlreichen klinischen Verpflichtungen in der Arbeitswoche (Montag bis Samstag zu Mittag), sind die Fellows verpflichtet an den Morgenfortbildungen jeden Dienstag, Donnerstag und Samstag um 07:00 Uhr teilzunehmen.

Der Schwerpunkt meines Fellowships war vorrangig im Gebiet der peripheren Nervenchirurgie. Meine Mentoren waren wie folgt:

Prof. Dr. Fu-Chan Wei, Pionier der Plastischen Chirurgie in Toe-To-Hand Transfer und Head \& Neck Rekonstruktion mit freiem mikrovaskulären Fibula- oder ALTLappenplastiken lehrte mir nicht nur seine Operationstechniken, sondern auch seine Gewohnheit vor dem Beginn jeder Operation am Whiteboard (Tafel) des Operationsaales mit Residents, Fellows und Visitors die Anamnese, Diagnose, In-

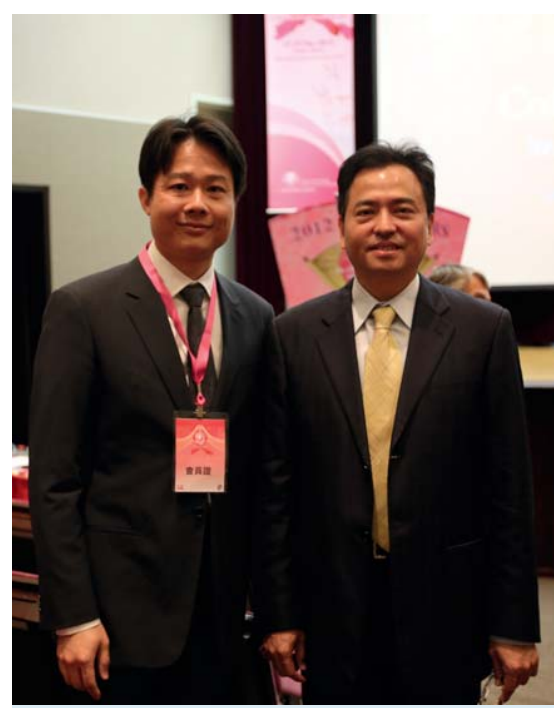

Abb. 5 Foto mit Prof. Dr. Ming-Huei Cheng (2012 Taipei International Breast Cancer Symposium, Taipei, Taiwan).

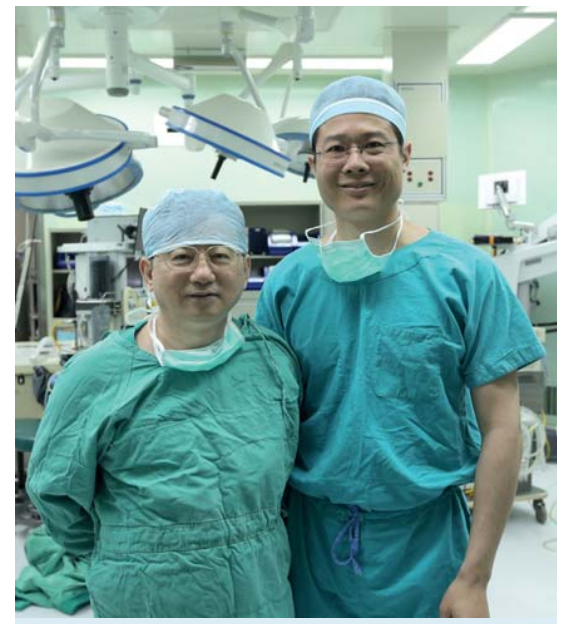

Abb. 6 Foto mit Prof. Dr. David Chwei-Chin Chuang im CGMH Linkou Operationssaal Nr. 3. dikation und den Therapieplan des aktuellen Patienten zu besprechen. Eine Gewohnheit, welche sehr hilfreich ist um den Operationsplan mit allen Beteiligten, inklusive dem resezierenden Operateur (HNO oder MKC) rückzusprechen und falls notwendig zu re-definieren. Prof. Wei lehrte mich seine Philosophie: „Be innovative and a pioneer in anything you do“. eine Lebenseinstellung welche ihm lebenslang mit Begeisterung begleitet hat (॰ Abb. 4).

Prof. Dr. Ming-Huei Cheng, ist Spezialist in Brustrekonstruktion mit freiem mikrovaskulären Bauchlappen und Vorreiter der freien mikrovaskulären Lymphknotenlappen. Vor allem mit Letzterem setzte er international neue Maßstäbe und eröffnete neue Wege in der chirurgischer Behandlung des Lymphödems. Durch sein kooperatives Engagement mit anderen Fachdisziplinen entstanden zahlreiche interdisziplinäre Publikationen. Nach seiner Wahl zum „Vice President“ in CGMH Linkou (2011) konnte er die fachübergreifende Zusammenarbeit der Plastischen Chirurgie in CGMH vertiefen. Prof. Cheng gab mir mit: „Cooperation is the key to success for today's plastic and reconstructive surgeon“ ( $\bullet$ Abb. 5).

Prof. Dr. David Chwei-Chin Chuang mit dem ich die meiste Zeit meines Fellowship verbracht habe, bildete mich in der peripheren Nervenchirurgie aus, insbesondere der Rekonstruktion der traumatischen und obstretischen Verletzungen des Plexus brachialis, als auch des kontralateralen C7 Spinalnerv Transfers und in der Reanimation des gelähmten Gesichts. Aufgrund des milden Klimas (subtropisch bis tropisch) besitzen in Taiwan ca. 60\% aller Fahrzeughalter eine Vespa (en.wikipedia.org/wiki/Motor cycle), welche auch der Grund von zahlreichen low-speed Motorradunfällen sind (www.chinapost.com.tw/taiwan/natio nal/national-news/2009/10/29/230674/ Official-denies.htm). Zweimal pro Woche sieht Prof. Chuang in den Sprechstunden bis zu zehn Neuvorstellungen von Armplexusverletzungen. Er ist stets bestrebt seinen Schülern zu motivieren mehr als er selbst zu erreichen und lehrte mich, „Think out of the box and surpass your teacher" (० Abb. 6).

Während des Fellowships war die klinische Arbeit körperlich anstrengend, da Operationen nicht selten bis in den späten Nachtstunden dauerten. Der organisatorische Ablauf im Spital war sehr effizient und es besteht eine harmonische Zusammenarbeit aller Berufsschichten. Es war nicht selten, dass sich das ganze 
Team nach der Arbeit privat zum gemeinsamen Abendessen traf um einerseits den langen Tag stimmig abzuschließen und andererseits sich für die gute Zusammenarbeit zu bedanken. Die Mentoren vergewisserten sich während den langen Operationen immer wieder, dass alle Mitglieder seines Teams die Mahlzeiten (mittags und abends) einnahmen.

Aufgrund der großen Anzahl an klinischen Fällen ist das CGMH nicht nur für die klinische Ausbildung sondern auch für wissenschaftliche Projekte sehr empfehlenswert. Meine wissenschaftlichen Projekte waren wegen des FWF Schrödinger Stipendiums mit Prof. Dr. David Chwei-Chin Chuang nervenchirurgisch gewichtet. In den Leerlaufphasen des FWF Projekts, konnte ich zusätzliche klinische und tierexperimentelle Projekte initiieren, betreuen und publizieren.

Wegen der großen internationalen Nachfrage ist es ratsam das Fellowship 2 Jahre im Voraus zu planen um auch zeitgerecht die entsprechende Finanzierung klären zu können. Möglichkeiten der Finanzierung wäre zum Beispiel: CGMH Fellowship Stipendium, ÖGPÄRCH Reisestipendium (www.plastischechirurgie.org), Forschungsstipendium der Österreichische Gesellschaft für Chirurgie (www.chirur gie-ges.at) und FWF Schrödinger Stipendium (www.fwf.ac.at/de/projects/schro edinger.html).

\section{Bekanntgabe $\nabla$}

Hiermit erklärt der Autor, dass er keine Interessenkonflikte mit den in diesem Bericht erwähnt Produkten hat. Der Autor hat nichts zu deklarieren.

(Disclosure: The author hereby declares that he has no conflict of interest in any products mentioned in this report and has nothing to declare.)

Dieses Fellowship und das gekoppelte wissenschaftliche Projekt wurden vom Erwin-Schrödinger- Stipendium (\# J3254B09) des Fonds zur Förderung der wissenschaftlichen Forschung (FWF) und vom Chang Gung Memorial Hospital Linkou Förderung unterstützt (CMRPG3B1211).

(Acknowledgements: This fellowship and its project were supported by the Erwin Schrödinger Fellowships scholarship (\# J3254-B09) of the Austrian Science Fund (FWF), awarded to the author (C.H.J. Tzou) and by a grant of the Chang Gung Memorial Hospital Linkou, Taiwan (CMRPG3B1211).

\section{Danksagungen \\ $\nabla$}

Mein Dank gebührt allen Mitarbeiter der CGMH Plastische und Rekonstruktive Chirurgie Linkou, insbesondere meinen Mentoren Prof. Dr. Fu-Chan Wei, Prof. Dr. Ming-Huei Cheng und Prof. David ChweiChin Chuang, als auch deren klinischen/ administrativen AssistentInen: Chen PeiJu, Chang Ruby Yih-Ru, Chiang Judy YuehYing, Chiang Wendy, Jaw Jill, Lo Tina
Yi-Hsuan, Tseng Bella Hsiao-Jung, und den zugeteilten Residents \& Attendings, die einen essentiellen Beitrag zu den Erfahrungen während des Fellowships leisteten: Dr. Chang Nai-Jen Tommy, Dr. Chen Jay Hsin-Hung, Dr. Chen Sirena Hsin-Yu, Dr. Hu Amber Jin-Hsuan, Dr. Lu Johnny Chuieng-Yi, Dr. Yang Shih-Yi und Dr. Yeh Dennis Hsuan-Keng.

Mein Dank in der Heimatinstitution gebührt: Univ.-Prof. Dr. Manfred Frey, Univ.Prof. Dr. Thomas Rath, Univ.-Prof. Dr. Oskar Aszmann, Univ.-Prof. Dr. Hildegunde Piza-Katzer und Univ.-Prof. Dr. Rafael L. Walzer.

\section{Präsentiert an der}

51. Jahrestagung der österreichischen Gesellschaft für Plastische, Ästhetische und Rekonstruktive Chirurgie 3.-5. Oktober 2013, Velden am Wörthersee, Österreich

Bibliografie

DOI http://dx.doi.org/

10.1055/s-0033-1361122

Handchir Mikrochir Plast Chir 2013; 45: 376-378

(c) Georg Thieme Verlag KG Stuttgart · New York ISSN 0722-1819

Korrespondenzadresse

Chieh-Han John Tzou, Dr. med

Klinische Abteilung für Plastische und

Rekonstruktive Chirurgie

Universitätsklinik für Chirurgie

Medizinisch Universität Wien, Österreich

Währinger Gürtel 18-20

1090 Wien

Österreich

chieh-han.tzou@meduniwien.ac.at 